\title{
Fast optical work-function tuning at an organic/metal interface
}

Wibke Bronsch, Daniel Przyrembel, Larissa Boie, Cornelius Gahl, and Martin Weinelt

Citation: Appl. Phys. Lett. 111, 081601 (2017);

View online: https://doi.org/10.1063/1.4999436

View Table of Contents: http://aip.scitation.org/toc/apl/111/8

Published by the American Institute of Physics

\section{Articles you may be interested in}

High resolution structural characterisation of laser-induced defect clusters inside diamond

Applied Physics Letters 111, 081103 (2017); 10.1063/1.4993118

High-resolution charge carrier mobility mapping of heterogeneous organic semiconductors Applied Physics Letters 111, 083302 (2017); 10.1063/1.4999762

Aluminum ultraviolet-visible plasmonic arrays for broadband and wavelength-selective enhancements of quantum dot emission

Applied Physics Letters 111, 081106 (2017); 10.1063/1.4986970

Exciting dynamic anapoles with electromagnetic doughnut pulses

Applied Physics Letters 111, 081104 (2017); 10.1063/1.4999368

Nitrogen-vacancy defects in diamond produced by femtosecond laser nanoablation technique Applied Physics Letters 111, 081101 (2017); 10.1063/1.4993751

Purcell-enhancement of the radiative PL decay in perylenediimides by coupling with silver nanoparticles into waveguide modes

Applied Physics Letters 111, 081102 (2017); 10.1063/1.4999325

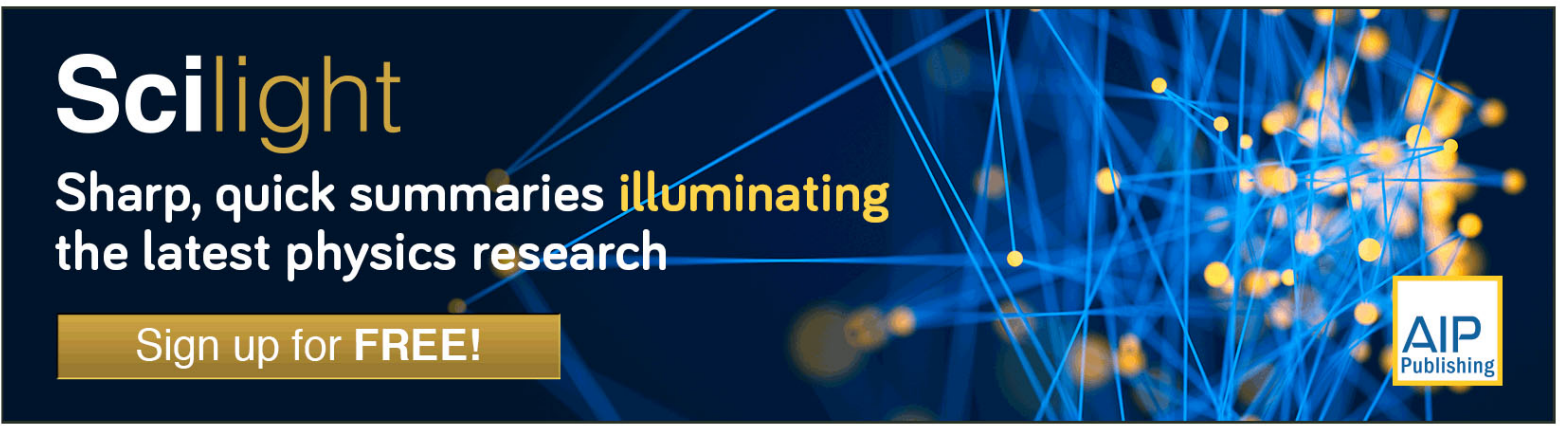




\title{
Fast optical work-function tuning at an organic/metal interface
}

\author{
Wibke Bronsch, Daniel Przyrembel, Larissa Boie, Cornelius Gahl, ${ }^{a)}$ and Martin Weinelt ${ }^{\text {a) }}$ \\ Freie Universität Berlin, Fachbereich Physik, Arnimallee 14, 14195 Berlin, Germany
}

(Received 8 April 2017; accepted 5 August 2017; published online 21 August 2017; corrected 24 August 2017)

\begin{abstract}
In a two-color experiment, we demonstrate how light can be used as an external control to continuously tune the work function of a gold substrate functionalized with a dilute azobenzene-based self-assembled monolayer (SAM). The work function is measured by two-photon photoelectron spectroscopy. While in the ground state the azobenzene moiety adopts the trans configuration, illumination with pulsed laser light at a wavelength of $368 \mathrm{~nm}$ results in a photostationary state (PSS) mainly comprising cis isomers. An additional $450 \mathrm{~nm}$ continuous-wave laser with tunable intensity serves to shift the PSS back towards the ground state. This way the work function is freely adjustable in real time over a range of $\sim 240 \mathrm{meV}$ between the two PSS extrema. We furthermore relate the change in work function to the average change in dipole moment per azobenzene chromophore. Quantum-chemical calculations that take into account available structural data of the molecules in the SAM must consider at least two different trans and four different cis orientations. The computed respective perpendicular trans-cis dipole-moment changes indicate that in experiment the cis molecules adopt different orientations along with a very high cis azobenzene yield in the UV PSS. Published by AIP Publishing. [http://dx.doi.org/10.1063/1.4999436]
\end{abstract}

The modification of inorganic materials with organic layers represents an active field of research that aims at overcoming material-class-specific drawbacks for device fabrication through the design of hybrid layered systems. Level alignment of electronic states at inorganic/organic interfaces is crucial in the fabrication of hybrid optoelectronic devices. ${ }^{1-3}$ One way to modify this alignment is to incorporate dipole layers into the interface. This results in changes of the work function at the surface. ${ }^{2-8}$ To extend the idea from a statically modified surface to a responsive surface or interface that is controllable by an external stimulus, the organic layers can be functionalized with molecular switches like the photochromic azobenzene. ${ }^{9-17}$ Its isomerization from the ground-state trans into the meta-stable cis form can be triggered by UV irradiation whereas illumination with blue light leads to back-switching. ${ }^{18,19}$ While the planar inversionsymmetric trans configuration has no static dipole moment, the bent $c$ is isomer is strongly polar. ${ }^{20}$ Stiller et al. used the impact of dipole-moment changes on the work function to prove the switching ability of different azobenzene derivatives in self-assembled monolayers (SAMs). ${ }^{10}$ However, only small changes of $20 \mathrm{meV}$ on a single-crystalline gold substrate were observed. Steric hindrance has been identified to prevent switching in densely packed SAMs, which stimulated the idea of chromophore dilution by insertion of additional alkanethiol molecules as lateral spacers..$^{19,21,22}$ SAMs from asymmetric azobenzene disulfides with a chromophore density of $50 \%$ showed work-function changes in the order of $200 \mathrm{meV} .{ }^{12}$ However, significant reductions in the maximal shift occurred for repeated UV irradiations. So far, in such experiments commonly photon fluxes in the order of $10^{16}$ photons $/ \mathrm{s} \mathrm{cm}^{2}$ or less were used, and hence, light-induced changes in the work function required several minutes to

\footnotetext{
${ }^{\text {a) }}$ Authors to whom correspondence should be addressed: c.gahl@fu-berlin.de and weinelt@physik.fu-berlin.de
}

attain a photostationary state (PSS). ${ }^{9-14,17}$ This would be too slow for useful applications. To drive the system into a desired PSS rapidly, we use the tunable interplay of laser light of two wavelengths corresponding to the $S_{1}$ and $S_{2}$ absorption bands of our azobenzene chromophore. ${ }^{19,23}$ This way we make the work function tunable in real time. A fspulsed laser beam at $368 \mathrm{~nm}$ (UV) triggers switching of the azobenzene units in the SAM from the trans into the cis form and simultaneously the emission of photoelectrons from the Au substrate via a two-photon process [see scheme in Fig. 1(a)]. These electrons pass through the tunable dipole field determined by the azobenzene trans-cis isomer ratio in the SAM and thereby probe the sample work function. In this way, we prevent beam damage to the SAM during the measurement, commonly caused by higher-energetic probe photons as, e.g., in ultraviolet photoelectron spectroscopy. To control the amount of cis isomers, we illuminate the same spot on our sample with an additional continuous-wave (cw) laser at $450 \mathrm{~nm}$ (blue). Its variable intensity is far too low to contribute to the two-photon photoemission (2PPE) signal by which the work function is measured. ${ }^{24}$ The isomerization cross sections $\sigma=1 /(j \cdot \tau)$ for both wavelengths are in the order of $10^{-18} \mathrm{~cm}^{2} .^{25}$ We used large photon fluxes $j$ in the order of $10^{17}-10^{19}$ photons per second and $\mathrm{cm}^{2}$, i.e., 10-1000 times more than in previous experiments, which allows driving the system into the PSSs within a time $5 \tau$ of just $10 \mathrm{~s}$ down to $0.5 \mathrm{~s}$.

The azobenzene-functionalized SAMs were prepared on a $2 \mathrm{~mm}$ thick $\mathrm{Au}(111)$ single crystal (MaTeck $\mathrm{GmbH}, 5 \mathrm{~N},<0.1^{\circ}$ ) by immersion as described elsewhere. ${ }^{19}$ The crystal surface was initially prepared by Ar ion bombardment and careful annealing. The work function $\Phi$ of the clean $\mathrm{Au}(111)$ surface was measured to be $5.4 \mathrm{eV}$, in agreement with the literature. ${ }^{26}$ Upon SAM formation, $\Phi$ is lowered by $\sim 1 \mathrm{eV}$, comparable to other works. ${ }^{27}$ Between SAM preparations, the crystal was cleaned with peroxomonosulfuric acid $\left[\mathrm{H}_{2} \mathrm{O}_{2}(30 \%): \mathrm{H}_{2} \mathrm{SO}_{4}(>95 \%)=1: 7 \mathrm{v} / \mathrm{v}\right]$ for 
a)
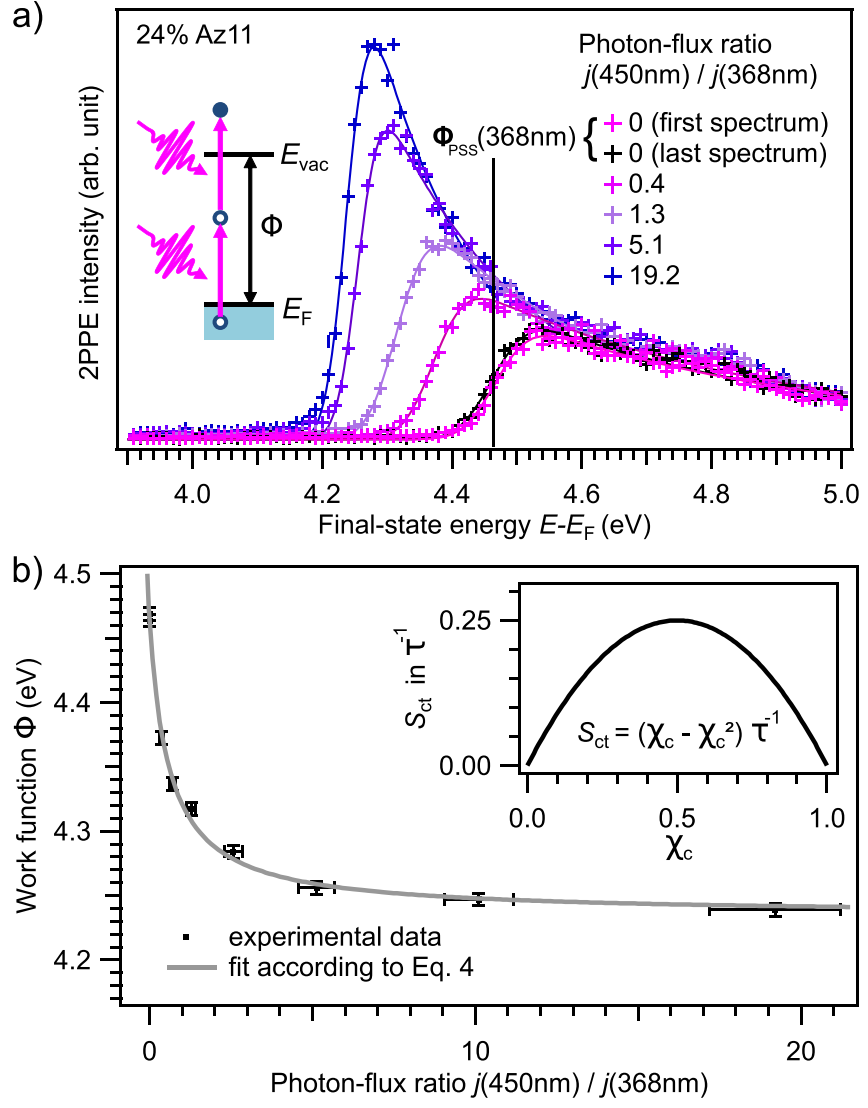

FIG. 1. (a) Select 2PPE spectra of a measurement series on a $24 \%$ Az11 SAM under different illumination conditions. The colored markers represent the measured data; the solid lines are fits to these data. The work function is determined from these fits as described in the text. Spectra for pure $368 \mathrm{~nm}$ irradiation in magenta and black: first and last spectra of the series. All other spectra are measured with additional $450 \mathrm{~nm}$ illumination. During the series, the photon flux of $368 \mathrm{~nm}$ was kept constant at $j(368 \mathrm{~nm})=(5.4 \pm 0.5) \cdot 10^{17} \mathrm{~s}^{-1} \mathrm{~cm}^{-2}$. The exposure time was $120 \mathrm{~s}$ per spectrum, $20 \mathrm{~min}$ for the whole series. Focal spot sizes: $(0.33 \pm 0.02) \mathrm{mm}^{2}$ at $450 \mathrm{~nm},(0.26 \pm 0.02) \mathrm{mm}^{2}$ at $368 \mathrm{~nm}$. (b) Sample work function from fits to the spectra shown in part (a) versus the ratio of the photon fluxes at 450 and $368 \mathrm{~nm}$. Inset: Switching events per second in a PSS as a function of the actual mole fraction of molecules in the cis state at the surface.

$10 \mathrm{~min}$ at room temperature and $20 \mathrm{~min}$ while heating to $60^{\circ} \mathrm{C}$. The methanolic immersion solutions contained 11-(4-(phenyldiazenyl)phenoxy)undecane-1-thiol (Az11, by courtesy of R. Klajn) and 1-dodecanethiol (C12, Alfa Aesar A12741), with an Az11 mole fraction of $20 \pm 3 \%$ (0.1 mM total thiol concentration). Co-adsorption of the two components yields densely packed SAMs containing $24 \pm 5 \%$ of Az11 molecules on the surface. ${ }^{19}$ To avoid premature photoswitching of the azobenzenes, preparation and experiments were performed under yellow light $(\lambda>500 \mathrm{~nm}$, Narva LT-T8 YELLOW special).

The ultrashort laser pulses at a wavelength of $368 \mathrm{~nm}$ were produced with a $300 \mathrm{kHz}$ Ti:Sa RegA-system (Coherent) pumping an optical-parametric amplifier in the visible regime and subsequent second-harmonic generation. Photoelectrons emitted from the grounded sample were detected with a time-of-flight spectrometer (SPECS Themis) in the drift mode. The work function is determined from fitting the low-energy cut-off in the 2PPE spectra with a model function. This is based on the product of an exponential decay with an error function centered at the energy of the vacuum level, i.e., the work function. The latter can be determined with a small relative uncertainty of $\sim 3 \mathrm{meV}$ within a series of measurements on the same sample spot. We used a diode laser (Oxxius) for the continuous-wave illumination at $450 \mathrm{~nm}$.

The pristine diluted Az11 SAM comprises azobenzene in trans configuration exclusively. ${ }^{19,25}$ Illumination at the $\mathrm{S}_{1}$ or $\mathrm{S}_{2}$ absorption bands triggers interconversion between the trans and cis isomers changing their ratio in the sample until a new steady state is reached. In this photostationary state, the switching rates in both directions become equal. For irradiation with $368 \mathrm{~nm}$ (inducing mainly trans-to-cis isomerization), we measure a work function of $\Phi_{\mathrm{PSS}}(368 \mathrm{~nm})=(4.465 \pm 0.003) \mathrm{eV}$ [cf. fit to the magenta data set in Fig. 1(a)]. Adding $450 \mathrm{~nm}$ light shifts the PSS back into the direction of the all-trans SAM. Note that different from the case of semiconducting substrates,${ }^{28}$ in the case of a metal substrate there is no contribution from the substrate to the photoinduced work-function shift. Figure 1(a) shows a series of spectra taken with different photon fluxes of the $450 \mathrm{~nm} \mathrm{cw}$ laser but a constant flux of the pulsed $368 \mathrm{~nm}$ laser of $j(368 \mathrm{~nm})=(5.4 \pm 0.5) \cdot 10^{17} \mathrm{~s}^{-1} \mathrm{~cm}^{-2}$. As soon as we add the cw laser, we observe a lowering of the work function. The work-function change can be related to the number of the cis isomers by the Helmholtz equation

$$
\Delta \Phi=\frac{e}{\epsilon_{0} \epsilon} \frac{N_{\mathrm{c}}}{A} \Delta p_{\perp},
$$

where $\frac{N_{\mathrm{c}}}{A}$ is the number of $c i s$-azobenzenes per unit area, $\Delta p_{\perp}$ the change in the perpendicular component of the dipole moment per chromophore, $\epsilon_{0}$ the vacuum permittivity, $\epsilon$ the relative permittivity of the organic layer, and $e$ the elementary electric charge. For the Az11 densities in the diluted SAM densities, depolarization effects can be neglected. ${ }^{29}$ Because in the PSS there is no net change in the number of cis molecules anymore, we can define the differential equation

$$
\dot{N}_{\mathrm{c}}=r_{\mathrm{tc}} \cdot N_{0}-\left(r_{\mathrm{tc}}+r_{\mathrm{ct}}\right) \cdot N_{\mathrm{c}} \stackrel{!}{=} 0,
$$

with $N_{0}=N_{\mathrm{c}}+N_{\mathrm{t}}$ being the total number of Az11 molecules and $r_{\mathrm{tc}}$ and $r_{\mathrm{ct}}$ being the rate constants of trans-to-cis (tc) and cis-to-trans (ct) isomerization, respectively. The rate constants $r_{\mathrm{tc}}=j_{\lambda 1} \sigma_{\mathrm{tc}}^{\lambda 1}+j_{\lambda 2} \sigma_{\mathrm{tc}}^{\lambda 2}$ and $r_{\mathrm{ct}}=j_{\lambda 1} \sigma_{\mathrm{ct}}^{\lambda 1}+j_{\lambda 2} \sigma_{\mathrm{ct}}^{\lambda 2}+r_{\mathrm{ct}}^{\mathrm{th}}$ depend on the respective photon fluxes $j$ and switching cross sections $\sigma$ in both directions for each wavelength, $\lambda 1$ $(368 \mathrm{~nm}, \mathrm{UV})$ and $\lambda 2(450 \mathrm{~nm}$, blue), and thermal backswitching into the trans state $r_{\mathrm{ct}}^{\text {th }}$. The latter can be neglected because it occurs on a time scale two orders of magnitude longer. ${ }^{25}$ Therefore, the work-function change, $\Phi_{\mathrm{PSS}}(x)-\Phi_{\mathrm{t}}$, can be written as

$$
\Delta \Phi(x)=\underbrace{\frac{e}{\epsilon_{0} \epsilon} \frac{N_{0}}{A} \Delta p_{\perp}}_{\Delta \Phi_{\max }}\left(\frac{\frac{\sigma_{\mathrm{tc}}^{\lambda 1}}{\sigma_{\mathrm{tc}}^{\lambda 2}}+x}{\frac{\sigma_{t c}^{\lambda 1}+\sigma_{\mathrm{ct}}^{\lambda 1}}{\sigma_{\mathrm{tc}}^{\lambda 2}}+x\left(\frac{\sigma_{\mathrm{tc}}^{\lambda 2}+\sigma_{\mathrm{ct}}^{\lambda 2}}{\sigma_{\mathrm{tc}}^{\lambda 2}}\right)}\right),
$$

with $x=\frac{j_{\lambda 2}}{j_{\lambda 1}}$ being the ratio of the two photon fluxes, ranging from zero to infinity. Thereby, the maximal work-function change between an all-cis and all-trans $\operatorname{SAM}, \Phi_{\mathrm{c}}-\Phi_{\mathrm{t}}$, is 
$\Delta \Phi_{\max }=\frac{\Phi_{\text {Pss }}(365 \mathrm{~nm})-\Phi_{\mathrm{t}}}{\chi_{\mathrm{c}}(365 \mathrm{~nm})}$, taking into account that the isomerization cis yield is $\chi_{\mathrm{c}}(\lambda)=\frac{\sigma_{\mathrm{tc}}^{\lambda}}{\sigma_{\mathrm{cc}}^{\lambda}+\sigma_{\mathrm{ct}}^{\lambda}}$. Figure $1(\mathrm{~b})$ shows the measured work function as a function of the ratio of the photon fluxes at $450 \mathrm{~nm}$ and $368 \mathrm{~nm}$. The grey line is a fit to the data according to

$$
\Phi_{\mathrm{PSS}}(x)=\Phi_{\mathrm{t}}+\Delta \Phi(x),
$$

using Eq. (3). In accordance with Moldt et al., the isomerization yield $\chi_{\mathrm{c}}(\lambda 2)$ at $\lambda 2=450 \mathrm{~nm}$ has been fixed to its maximum value of $5 \%$ in the fit shown in Fig. 1(b) ${ }^{25}$ With that, Eq. (4) describes the measured work-function change very well. Given its derivation from first-order kinetics, assuming that the azobenzene moieties act independent of each other, there seems to be no large cooperative-switching effect in the diluted SAM. The fit gives us the work function of the all-trans SAM $\Phi_{\mathrm{t}}=(4.22 \pm 0.01) \mathrm{eV}$. For large $450 \mathrm{~nm}$ photon fluxes, the work function approaches a limiting value of $\Phi_{\text {PSS }}(450 \mathrm{~nm})=(4.23 \pm 0.01) \mathrm{eV}$ due to residual cis isomers. Thus, the maximal work-function shift with respect to the UV PSS (368 nm only) is $\Delta \Phi_{\mathrm{PSS}}(368 \mathrm{~nm} \Longleftrightarrow 450 \mathrm{~nm}) \approx(240 \pm 10) \mathrm{meV}$. With this, we can determine the average change in dipole moment perpendicular to the surface per azobenzene unit. By means of atomic force microscopy, the molecular density in a pure Az11 SAM on $\mathrm{Au}(111)$ has been found to be $(4.1 \pm 0.3) \cdot 10^{14} \mathrm{~cm}^{-2} \cdot{ }^{30}$ In our diluted SAM, this translates to a density of $(9.8 \pm 0.7) \cdot 10^{13} \mathrm{~cm}^{-2}$. (The $10 \%$ larger unit mesh determined by scanning tunneling microscopy ${ }^{31}$ would enlarge the calculated dipole-moment change by $10 \%$ as well.) The relative permittivity of azobenzene-functionalized alkanethiols in pure and mixed SAMs has been studied by surface plasmon resonance (SPR) spectroscopy. ${ }^{13}$ Considering those results, we assume a relative permittivity $\epsilon=2.5 \pm 0.2$ for our samples. Using these values, our observed work-function change necessitates an average dipole-moment change $\Delta p_{\perp}=1.6 \pm 0.4 \mathrm{D}$ per Az11 molecule in the SAM.

According to a near edge $\mathrm{X}$-ray absorption fine structure (NEXAFS) study, ${ }^{25}$ the isomerization yield of $365 \mathrm{~nm}$ irradiation $\chi_{\mathrm{c}}(365 \mathrm{~nm})$ is in the range of $67 \%-100 \%$ in the case of an Az11 coverage of $24 \%$. This corresponds to an average difference in dipole moment perpendicular to the surface of $(2.4 \pm 0.6)$ to $(1.6 \pm 0.4) \mathrm{D}$ between cis and trans Az11. This result seems to be in agreement with the value of $2.04 \mathrm{D}$ per azobenzene unit that Nagahiro et al. ${ }^{13}$ derived from density functional theory (DFT) calculations for 1:1 mixed SAMs of Az12 and C12 formed from the respective asymmetric disulfide. However, newer results from NEXAFS and UV/Vis spectroscopy indicate that the average orientation of Az11 with its one-methylene-unit shorter linker chain strongly differs from the one those calculations are based on. ${ }^{19,32-34}$ The NEXAFS polarization contrast of a pure C12 SAM indicates that on average the alkyl chains are canted away from the surface normal by $\vartheta_{\mathrm{CC}} \approx 38^{\circ}$ and the methylene groups by $\alpha_{\mathrm{CH} 2} \approx 63^{\circ}$ (cf. Fig. 2). This implies that the carbon-atom backbone is twisted by a roll angle of $\gamma_{\mathrm{C} 12} \approx 42.5^{\circ}$. These findings are in fair agreement with the orientation of alkanethiols on $\mathrm{Au}(111)$ determined by vibrational spectroscopy $(\vartheta$
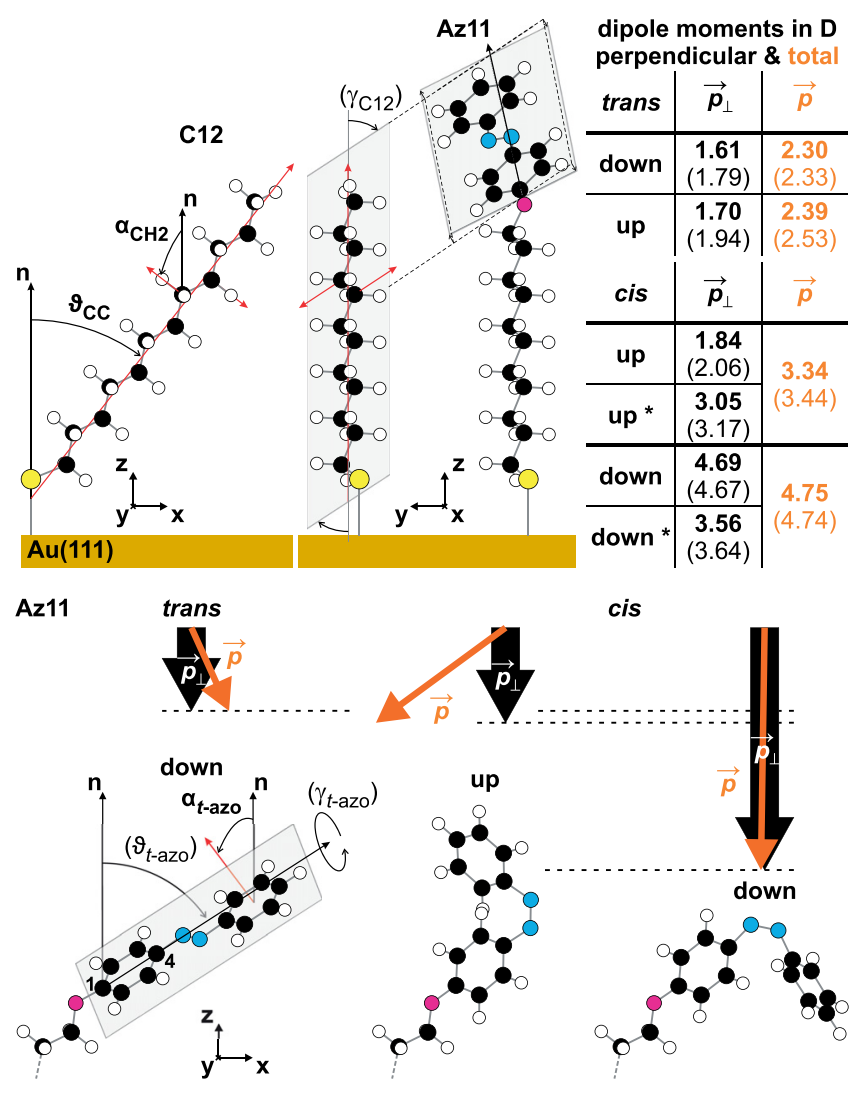

FIG. 2. Top left/middle: Schematic of the NEXAFS-compatible, DFT-optimized orientations of the $\mathrm{C} 12$ spacer and trans-Az11. Top right: dipole moments perpendicular to the surface and total from MP2/6-311++G(d,p)// B3LYP/6-311++G(d,p) calculations (B3LYP/6-311++G(d,p) values for reference). Bottom: Schematic of the trans down and cis up and down isomer orientations along with projections of their respective dipole-moment vectors on the $\mathrm{x}-\mathrm{z}$ plane. The respective mirror images $(*)$ of the cis isomers by reflection through the alkyl-linker chain plane are not shown. The orientations of the NEXAFS transition-dipole moments at the methylene (CH2) groups, the alkyl linker chain (CC), and the phenyl-ring planes are indicated by red arrows.

$\left.\approx 27^{\circ}, \gamma \approx 53^{\circ}\right) .{ }^{35}$ NEXAFS measurements on a $100 \%$ Az1 1 SAM give average orientations of the trans-azobenzene plane vectors, i.e., perpendicular to the phenyl-ring planes and the azo group, of $\alpha_{t \text {-azo }} \approx 72^{\circ}$ with respect to the surface normal. This indicates a tilt angle of the chromophore $\mathrm{C} 1-\mathrm{C} 4$ axis of $\vartheta_{t \text {-azo }} \approx 30^{\circ}$ from the normal (cf. Fig. 2) ${ }^{19,25}$ Furthermore, the orientation of the $\mathrm{N}=\mathrm{N}$ double bond is rather parallel to the surface. ${ }^{19}$ The orientation of the chromophores depends on their surface density: Upon dilution in mixed SAMs of Az11 and $\mathrm{C} 12$, the azobenzene moiety tilts down towards the surface plane. In the case of an Az11 surface concentration of ca. $15 \%$, the mean orientation of the azobenzene plane vectors assumes just $\alpha_{t \text {-azo }}=(45 \pm 5)^{\circ}$ from normal which translates to $\vartheta_{t \text {-azo }} \geq 45^{\circ} .{ }^{19}$ The orientations of the transition dipole moments are depicted as red arrows in Fig. 2.

Assuming that all molecules adopt an orientation with the average angles deduced from NEXAFS, we calculated possible orientations of Az11 and resulting dipole moments. The matrix of the surrounding $\mathrm{C} 12$ spacer chains sets the orientation of the alkyl linker chain of Az11 which implies $\alpha_{t \text {-azo }} \approx 65^{\circ}$. We established angles $\alpha_{t \text {-azo }}=45^{\circ}$ in the diluted SAM by an additional intramolecular twist of ca. $25^{\circ}$ about the oxygen-phenyl bond. At room temperature, roll angles 
on this order around the equilibrium orientation of this bond should readily occur. ${ }^{34}$ Interactions with neighboring chromophores and the surrounding SAM surface could further facilitate this twisting. The azo group can point either away from or towards the surface giving two possible orientations of the trans-chromophore, denoted as trans up and trans down. For the cis isomer, very little is known from experiment about its orientation in a SAM. Our NEXAFS measurements show minor polarization dependence pointing towards various orientations of the chromophore. ${ }^{25}$ Most likely, the lower phenyl ring takes on an equilibrium orientation with respect to the attached alkyl linker chain. The upper phenyl ring then can move either towards or away from the surface upon switching from the trans into the cis form. This leads to different $c i$ s orientations, again with the azo group pointing rather down or up with respect to the surface. The tilt and roll angles of the alkyl linker chain at the surface lift the equivalence of the "down" and "up" cisAz11 isomers with their respective mirror images (*). That is why we have to consider two different trans and four different cis orientations of the chromophores. To determine the dipole-moment changes perpendicular to the surface whilst taking into account the mentioned geometrical constraints, we carried out quantum-chemical calculations with density functional theory (DFT, B3LYP ${ }^{36,37}$ ) and ab-initio perturbation theory $\left(\mathrm{MP} 2^{38}\right)$, both at the $6-311++\mathrm{G}(\mathrm{d}, \mathrm{p})$ level. We first optimized the four possible equilibrium geometries of individual trans- and cis-Az11 isomers in vacuum by DFT with the alkyl linker chain constrained to an overall straight zigzag arrangement. Dipole moments of all isomers were calculated by MP2 using the DFT-optimized structures. ${ }^{39}$ The values are given in the table in Fig. 2. For the trans isomer, the direction of the azo group (up or down) has a weak influence on its dipole moment, changing it by less than $6 \%$. Also the intramolecular twist of the chromophore has a rather negligible effect. This is reasonable because the dipole moment of trans-Az11 is mainly determined by the angle and orientation of the COC ether bonds bridging the linker chain and the highly symmetrical flat chromophore. In the cis isomer however, the bent azobenzene moiety adds a strong dipole moment whose direction in turn depends on the orientation of the azo group. In the diluted Az11 SAM, the tilt and roll imposed by the alkyl linker chain determine the orientation of the azo group which varies between nearly perpendicular (cis up) and parallel (cis down) to the surface. Thereby, the dipolemoment component of the cis-azobenzene unit varies between nearly parallel and perpendicular to the surface, respectively. This makes for very different perpendicular cis-Az11 dipolemoment components. Therefore, the perpendicular dipolemoment changes vary between very small for when the azo group is perpendicular (cis up) $\Delta p_{\perp} \simeq 0.18 \mathrm{D}$ and very large for when it is parallel (cis down) $\Delta p_{\perp} \simeq 3.03 \mathrm{D}$ to the surface. In the experimental UV PSS, the cis up state clearly cannot be the exclusive one and the sterically more demanding cis down state is unlikely to be predominant. In the case of an even distribution of the possible cis isomers, the average change in dipole moment between the UV PSS and the $450 \mathrm{~nm}$ limit would be $\Delta p_{\perp} \simeq 1.63$ D. Statistical mixing of Az11 and C12 leads to different local environments for the individual chromophores.
Therefore, most likely all four cis orientations should be present at the surface.

Finally, we address the reversibility of the switching process under the experimental conditions. Figure 1(a) shows two spectra taken with only $368 \mathrm{~nm}$ on the same sample spot at the beginning and the end of the spectra series. The number of times each Az11 molecule switched during the series can be estimated on the basis of Eq. (2). In a PSS, equal numbers of molecules switch in each direction. Hence, the number of switching cycles $S_{\circlearrowleft}$ equals the number of switching events in one direction $S_{\mathrm{ct}}=\frac{r_{\mathrm{ct}} \cdot N_{\mathrm{c}}}{N} \stackrel{!}{=} S_{\circlearrowleft}$. This quantity can be expressed as a function of the isomerization yield $\chi_{\mathrm{c}}=\frac{N_{\mathrm{c}}}{N}=\frac{r_{\mathrm{tc}}}{r_{\mathrm{tc}}+r_{\mathrm{ct}}}$ of a specific wavelength or wavelength combination and the sum of the rate constants which equals the inverse rise time $\frac{1}{\tau}$ to reach a PSS

$$
S_{\circlearrowleft}=\left(\chi_{\mathrm{c}}-\chi_{\mathrm{c}}^{2}\right) \cdot\left(r_{\mathrm{ct}}+r_{\mathrm{tc}}\right)=\left(\chi_{\mathrm{c}}-\chi_{\mathrm{c}}^{2}\right) \frac{1}{\tau} .
$$

Thus, the number of switching cycles depends strongly on the isomerization yield, as shown in the inset of Fig. 1(b). Taking the isomerization cross sections $\sigma_{\mathrm{tc}}$ and $\sigma_{\mathrm{ct}}$ for $365 \mathrm{~nm}$ and $455 \mathrm{~nm}$ determined by NEXAFS measurements ${ }^{25}$ and interpolating the isomerization yields that are reached for the different photon flux ratios by the measured work functions, we find that each molecule can undergo more than 800 individual switching events in our measurement series without significant fatigue.

In conclusion, diluted azobenzene-functionalized alkanethiol SAMs allow fast, reversible, and continuous tuning of the work function of gold electrodes over a range of more than $200 \mathrm{meV}$ via external stimulation by light. This range may be significantly extended by adding polar end-groups that enlarge the perpendicular dipole-moment change. ${ }^{13}$ The potential of this system for future applications is not only given by the tunable range but also by its fast response and low fatigue. Even after several hundred switching events of every molecule, the work function is precisely reproduced. Our calculations clearly indicate the importance of considering molecular orientations close to experiment to get reasonable results. In our case, this necessitates evaluating perpendicular dipole-moment components for six different cases-two trans and four cis orientations. The observed maximum trans-cis dipole-moment change agrees very well with the average of the calculated ones. This strongly hints that there is not one defined cis orientation in the UV PSS but that in the ensemble we obtain an average one. The seemingly non-cooperative switching between trans and cis might be related to the lack of one defined cis state in which then defined intermolecular interactions could come into play. For applications, the next step would be to not only control the switching but also the orientation of the azobenzene unit in the cis form. This asks for the design of specific photoswitches or also constraining the possible molecular movements upon switching by modeling the SAM environment accordingly.

The authors gratefully acknowledge financial support by the DFG through Sfb 658 and the Helmholtz VI 419 and the kind supply of Az11 through Rafal Klajn from the Weizmann Institute of Science, Israel. 
${ }^{1}$ N. Crivillers, E. Orgiu, F. Reinders, M. Mayor, and P. Samorì, Adv. Mater. 23, 1447 (2011).

${ }^{2}$ M. Oehzelt, K. Akaike, N. Koch, and G. Heimel, Sci. Adv. 1, e1501127 (2015).

${ }^{3}$ T. Schultz, R. Schlesinger, J. Niederhausen, F. Henneberger, S. Sadofev, S. Blumstengel, A. Vollmer, F. Bussolotti, J.-P. Yang, S. Kera, K. Parvez, N. Ueno, K. Müllen, and N. Koch, Phys. Rev. B 93, 125309 (2016).

${ }^{4}$ R. W. Zehner, B. F. Parsons, R. P. Hsung, and L. R. Sita, Langmuir 15, 1121 (1999).

${ }^{5}$ D. M. Alloway, M. Hofmann, D. L. Smith, N. E. Gruhn, A. L. Graham, R. J. Colorado, V. H. Wysocki, T. R. Lee, P. A. Lee, and N. R. Armstrong, J. Phys. Chem. B 107, 11690 (2003).

${ }^{6}$ B. de Boer, A. Hadipour, M. M. Mandoc, T. van Woudenbergh, and P. W. M. Blom, Adv. Mater. 17, 621 (2005).

${ }^{7}$ V. De Renzi, R. Rousseau, D. Marchetto, R. Biagi, S. Scandolo, and U. del Pennino, Phys. Rev. Lett. 95, 046804 (2005).

${ }^{8}$ D. A. Egger, F. Rissner, G. M. Rangger, O. T. Hofmann, L. Wittwer, G. Heimel, and E. Zojer, Phys. Chem. Chem. Phys. 12, 4291 (2010).

${ }^{9}$ D. Gustina, E. Markava, I. Muzikante, B. Stiller, and L. Brehmer, Adv. Mater. Opt. Electron. 9, 245 (1999).

${ }^{10}$ B. Stiller, G. Knochenhauer, E. Markava, D. Gustina, I. Muzikante, P. Karageorgiev, and L. Brehmer, Mater. Sci. Eng., C 8-9, 385 (1999).

${ }^{11}$ B. Stiller, P. Karageorgiev, T. Jüngling, D. Prescher, T. Zetzsche, R. Dietel, G. Knochenhauer, and L. Brehmer, Mol. Cryst. Liq. Cryst. 355, 401 (2001).

${ }^{12}$ L. F. N. Ah Qune, H. Akiyama, T. Nagahiro, K. Tamada, and A. T. S. Wee, Appl. Phys. Lett. 93, 083109 (2008).

${ }^{13}$ T. Nagahiro, H. Akiyama, M. Hara, and K. Tamada, "Characterization of self-assembled monolayers by advanced spectroscopic techniques," J. Electron Spectrosc. Relat. Phenom. 172, 128 (2009).

${ }^{14}$ N. Crivillers, A. Liscio, F. Di Stasio, C. Van Dyck, S. Osella, D. Cornil, S. Mian, G. M. Lazzerini, O. Fenwick, E. Orgiu, F. Reinders, S. Braun, M. Fahlman, M. Mayor, J. Cornil, V. Palermo, F. Cacialli, and P. Samorì, Phys. Chem. Chem. Phys. 13, 14302 (2011).

${ }^{15}$ E. Orgiu, N. Crivillers, M. Herder, L. Grubert, M. Pätzel, J. Frisch, E. Pavlica, D. T. Duong, G. Bratina, A. Salleo, N. Koch, S. Hecht, and P. Samorì, Nat. Chem. 4, 675 (2012).

${ }^{16}$ N. Heinemann, J. Grunau, T. Leißner, O. Andreyev, S. Kuhn, U. Jung, D. Zargarani, R. Herges, O. Magnussen, and M. Bauer, Chem. Phys. 402, 22 (2012).

${ }^{17}$ N. Crivillers, S. Osella, C. Van Dyck, G. M. Lazzerini, D. Cornil, A. Liscio, F. Di Stasio, S. Mian, O. Fenwick, F. Reinders, M. Neuburger, E. Treossi, M. Mayor, V. Palermo, F. Cacialli, J. Cornil, and P. Samorì, Adv. Mater. 25, 432 (2013).
${ }^{18}$ E. Fischer, M. Frankel, and R. Wolovsky, J. Chem. Phys. 23, 1367 (1955).

${ }^{19}$ T. Moldt, D. Brete, D. Przyrembel, S. Das, J. R. Goldman, P. K. Kundu, C. Gahl, R. Klajn, and M. Weinelt, Langmuir 31, 1048 (2015).

${ }^{20}$ G. S. Hartley and R. J. W. Le Fevre, J. Chem. Soc. 1939, 531 (1939).

${ }^{21}$ A. S. Kumar, T. Ye, T. Takami, B.-C. Yu, A. K. Flatt, J. M. Tour, and P. S. Weiss, Nano Lett. 8, 1644 (2008).

${ }^{22}$ D. T. Valley, M. Onstott, S. Malyk, and A. V. Benderskii, Langmuir 29, 11623 (2013).

${ }^{23}$ J. T. Foy, Q. Li, A. Goujon, J.-R. Colard-Itté, G. Fuks, E. Moulin, O. Schiffmann, D. Dattler, D. P. Funeriu, and N. Giuseppone, Nat. Nanotechnol. 12, 540-545 (2017).

${ }^{24}$ T. Fauster, H. Petek, and M. Wolf, "Surface states and absorbate-induced electronic structure," in Dynamics at Solid State Surfaces and Interfaces, Volume 2: Fundamentals, edited by U. Bovensiepen, H. Petek, and M. Wolf (Wiley-VCH, 2012), Chap. 3, pp. 115-154.

${ }^{25}$ T. Moldt, D. Przyrembel, M. Schulze, W. Bronsch, L. Boie, D. Brete, C. Gahl, R. Klajn, P. Tegeder, and M. Weinelt, Langmuir 32, 10795 (2016).

${ }^{26}$ H. B. Michaelson, J. Appl. Phys. 48, 4729 (1977).

${ }^{27}$ M. Muntwiler, C. D. Lindstrom, and X. Y. Zhu, J. Chem. Phys. 124, 081104 (2006).

${ }^{28}$ I. V. Malyar, E. Titov, N. Lomadze, P. Saalfrank, and S. Santer, J. Chem. Phys. 146, 104703 (2017).

${ }^{29}$ O. L. A. Monti and M. P. Steele, Phys. Chem. Chem. Phys. 12, 12390 (2010).

${ }^{30}$ M. Jaschke, H. Schönherr, H. Wolf, H.-J. Butt, E. Bamberg, M. K. Besocke, and H. Ringsdorf, J. Phys. Chem. 100, 2290 (1996).

${ }^{31}$ S. C. B. Mannsfeld, T. W. Canzler, T. Fritz, H. Proehl, K. Leo, S. Stumpf, G. Goretzki, and K. Gloe, J. Phys. Chem. B 106, 2255 (2002).

${ }^{32}$ D. Gnatek, S. Schuster, J. Ossowski, M. Khan, J. Rysz, S. Krakert, A. Terfort, M. Zharnikov, and P. Cyganik, J. Phys. Chem. C 119, 25929 (2015).

${ }^{33}$ D. Brete, D. Przyrembel, C. Eickhoff, R. Carley, W. Freyer, K. Reuter, C. Gahl, and M. Weinelt, J. Phys.: Condens. Matter 24, 394015 (2012).

${ }^{34}$ C. Gahl, R. Schmidt, D. Brete, E. R. McNellis, W. Freyer, R. Carley, K. Reuter, and M. Weinelt, J. Am. Chem. Soc. 132, 1831 (2010).

${ }^{35}$ P. E. Laibinis, G. M. Whitesides, D. L. Allara, Y. T. Tao, A. N. Parikh, and R. G. Nuzzo, J. Am. Chem. Soc. 113, 7152 (1991).

${ }^{36}$ A. D. Becke, J. Chem. Phys. 98, 5648 (1993).

${ }^{37}$ P. J. Stephens, F. J. Devlin, C. F. Chabalowski, and M. J. Frisch, J. Phys. Chem. 98, 11623 (1994).

${ }^{38}$ C. Møller and M. S. Plesset, Phys. Rev. 46, 618 (1934).

${ }^{39}$ M. Schulze, M. Utecht, T. Moldt, D. Przyrembel, C. Gahl, M. Weinelt, P. Saalfrank, and P. Tegeder, Phys. Chem. Chem. Phys. 17, 18079 (2015). 